\title{
Pacific
}

Journal of

Mathematics

\section{ON THE SUM OF POWERED DISTANCES \\ TO CERTAIN SETS OF POINTS ON THE CIRCLE}

Nikolai Nikolov and Rafael RAFAilov

Volume $253 \quad$ No. 1

September 2011 


\title{
ON THE SUM OF POWERED DISTANCES TO CERTAIN SETS OF POINTS ON THE CIRCLE
}

\author{
NiKOlai NiKOLOV AND RAFAEL RAFAILOV
}

\begin{abstract}
We consider an extremal problem in geometry. Let $\lambda$ be a real number and let $A, B$ and $C$ be arbitrary points on the unit circle $\Gamma$. We give a full characterization of the extremal behavior of the function $f(M, \lambda)=$ $M A^{\lambda}+M B^{\lambda}+M C^{\lambda}$, where $M$ is a point on the unit circle as well. We also investigate the extremal behavior of $\sum_{i=1}^{n} X P_{i}$, where the $P_{i}$, for $i=1, \ldots, n$, are the vertices of a regular $n$-gon and $X$ is a point on $\Gamma$, concentric to the circle circumscribed around $P_{1} \ldots P_{n}$. We use elementary analytic and purely geometric methods in the proof.
\end{abstract}

\section{Introduction}

The question of placing electrical charges on a sphere in such a way that the potential energy of the system obtains its extremal values has long been of importance to physics. Problems of this kind have also been considered in classical potential theory.

The planar case of this question is answered by the general solution of placing $n$ points $M_{i}, i=1, \ldots, n$ on the unit circle in such a way as to obtain the extreme values of the sum

$$
\sum_{0 \leq i<j \leq n}\left|M_{i} M_{j}\right|^{\lambda}
$$

where $\lambda$ is a given real number, the concrete case being $\lambda=-1$. (We denote the distance between two points $P$ and $Q$ by $|P Q|$, or by $P Q$ when there is no danger of confusion.)

There is a growing amount of literature on this problem, which we can regard as a discrete analog of questions studied in classical potential theory. This has led to the development of the problem of placing a point $M$ on the unit circle in such a way to obtain the extremal values of

$$
\sum_{i=1}^{n} M M_{i}^{\lambda}
$$

MSC2010: 52A40.

Keywords: powered distance, unit circle. 
for a given point set $M_{i}, i=1, \ldots, n$. This has proven to be a difficult question and in general it remains open.

Here we consider the case $n=3$ and prove:

Theorem 1.1. Let $\lambda$ be a real number and let $A, B$ and $C$ be arbitrary points on the unit circle $\Gamma$.

(1) If $\lambda<0$, there is always a point $M$ on $\Gamma$ such that $f(M, \lambda) \leq 2+2^{\lambda}$.

(2) If $\lambda \in[0 ; 2]$, there is always a point $M$ on $\Gamma$ such that $f(M, \lambda) \geq 2+2^{\lambda}$.

(3) If $\lambda \in(2 ; 4)$, there is always a point $M$ on $\Gamma$ such that $f(M, \lambda) \geq 2 \sqrt{3} \lambda$.

(4) If $\lambda \geq 4$, there is always a point $M$ on $\Gamma$ such that $f(M, \lambda) \geq 2+2^{\lambda}$.

These bounds are sharp if and only if $A, B$ and $C$ are the vertices of an equilateral triangle.

Or, equivalently,

(1) $\min _{A, B, C \in \Gamma} \max _{M \in \Gamma} f(M, \lambda)=2+2^{\lambda}$ for $\lambda \in[0 ; 2] \cup[4 ; \infty)$.

(2) $\min _{A, B, C \in \Gamma} \max _{M \in \Gamma} f(M, \lambda)=2 \sqrt{3}^{\lambda}$ for $\lambda \in(2 ; 4)$.

(3) $\max _{A, B, C \in \Gamma} \min _{M \in \Gamma} f(M, \lambda)=2+2^{\lambda}$ for $\lambda<0$.

In the last case, the order of the maximum and the minimum is reversed. We are not interested in the maximum of the function $f(M, \lambda)$ when $\lambda<0$, since it is infinity when $M$ approaches $A, B$ or $C$.

Prior to the present article, the exact extremal values of $f(M, \lambda)$, established in [Stolarsky 1975], were only known for $\lambda \in[0 ; 2]$. We also give another (more elementary) proof of the results obtained in that article.

The question of the extremal behavior of the function

$$
f_{n}(M,-2)=\sum_{i=1}^{n} M M_{i}^{-2}
$$

is considered in [Ambrus and Ball 2011], where it is proved that there always exists a point $M \in \Gamma$ such that

$$
f_{n}(M,-2) \leq \frac{1}{4} n^{2} .
$$

This bound is sharp if and only if the $M_{i}$ are the vertices of a regular $n$-gon. This agrees with our results when $n=3$.

We also consider the case when there are $n$ points $P_{i}, i=1, \ldots, n$ on the unit circle that are the vertices of a regular $n$-gon, and a point $X$ on a circle $\Gamma$, concentric to the circumscribed circle of $P_{1} \ldots P_{n}$. We study the extremal values of

$$
R_{n}(X, \lambda)=\sum_{i=1}^{n} X P_{i}^{\lambda},
$$

where $X \in \Gamma$. 
This problem was considered by Stolarsky [1975], who solved it for $0 \leq \lambda<2 n$, where $\Gamma$ is the circumscribed circle of the polygon, and by Mushkarov [1982], who found $\lambda$ for which the sum does not depend on the position of $X$ on $\Gamma$, again where $\Gamma$ is circumscribed around $P_{1} \ldots P_{n}$, and gave a trigonometric representation for higher powers. In this paper we characterize the extremal behavior of the sum $\sum_{i=1}^{n} X P_{i}^{\lambda}$ and prove:

Theorem 1.2. Let $P_{i}, i=1, \ldots, n$ be the vertices of a regular $n$-gon inscribed in the unit circle. Now let $\Gamma$ be a circle concentric to the circumscribed circle. Put $B_{i}=O P_{i} \cap \Gamma$, where $O$ is the center of the $n$-gon.

Let $X \in \Gamma$ and

$$
R_{n}(X, \lambda)=\sum_{i=1}^{n} X P_{i}^{\lambda}
$$

(1) Suppose $\lambda<0$. The minimum of $R_{n}(X, \lambda)$ is achieved when $X$ bisects the arc between consecutive vertices of $B_{1} \ldots B_{n}$, and the maximum when $X \equiv B_{i}$. In the case when $\Gamma$ is the circumscribed circle around $P_{1} \ldots P_{n}$, this function is not bounded when $X \rightarrow B_{i}$ for some $i$.

(2) Suppose $0 \leq \lambda<2 n$. If $\lambda$ is an even integer, then $R_{n}(X, \lambda)$ is independent of the position of $X$ on $\Gamma$.

Otherwise let $m$ be such an integer that $2 m \leq \lambda \leq 2 m+2$.

If $m$ is even (resp. odd), then $R_{n}(X, \lambda)$ is maximal (resp. minimal) if and only if $X$ bisects the arc between consecutive vertices of $B_{1} \ldots B_{n}$. Also $R_{n}(X, \lambda)$ is minimal (resp. maximal) if and only if $M \equiv B_{i}$.

(3) $2 n \leq \lambda$. If $n$ is even (resp. odd), the maximum (resp. minimum) of $R_{n}(X, \lambda)$ is obtained when $X$ coincides with one of the vertices of $B_{1} \ldots B_{n}$, and the minimum (resp. maximum) is achieved when $X$ bisects the arc between consecutive vertices.

Remark 1.3. A case of conclusion (2) of the theorem is proved in [Stolarsky 1975], when $\Gamma$ is the circle circumscribed around $P_{1} \ldots P_{n}$. However, it seems possible that the general result of (2) can be proved in the same manner.

It is easy to see that (3) is actually true for $\lambda>2 n-2$.

We begin by considering the regular $n$-gon, since we use the result later.

\section{The regular $n$-gon}

We say that $y$ is a root of degree $k$ of an equation $f(x)=0$, where $f$ is $k$-times differentiable, if $f(y)=0$ and $f^{t}(y)=0$ for $t=1, \ldots, k-1$ and $f^{k}(y) \neq 0$, where $f^{t}(x)$ denotes the $t$-th derivative of $f$. 
Lemma 2.1. Let $a_{1}, a_{2}, \ldots, a_{n}$ be real numbers and $b_{1}, b_{2}, \ldots, b_{n}$ be nonnegative numbers. Then the function

$$
\varphi(\lambda)=\sum_{i=1}^{n} a_{i} b_{i}^{\lambda}
$$

is either identically zero or has at most $n-1$ real zeros counted with their multiplicities.

Proof. We proceed by induction on the number of summands. For $n=1$, we have $a b^{\lambda}=0$, which does not have solutions if both $a$ and $b$ are nonzero. If either of them is zero, then $a b^{\lambda}$ is identically zero. Now assume the statement to be true for all $k<n$. For $k=n$, if either of $a_{i}$ or $b_{i}$ is zero, then we use the induction hypothesis.

Now let $b_{i}, a_{i}$ be nonzero. Since all of $b_{i}$ are nonzero, we can divide each term by $b_{1}^{\lambda}$ to get

$$
\sum_{i=1}^{n} a_{i}\left(\frac{b_{i}}{b_{1}}\right)^{\lambda}=0 .
$$

Assume that this equation is not identically zero, and that its solutions are $y_{1}, \ldots, y_{k}$ with multiplicities $t_{1}, \ldots, t_{k}$ and $\sum_{i=1}^{k} t_{i}>n-1$.

Differentiating this with respect to $\lambda$, we get

$$
\sum_{i=2}^{n} a_{i} \ln \frac{b_{i}}{b_{1}}\left(\frac{b_{i}}{b_{1}}\right)^{\lambda}=0=\sum_{i=2}^{n} a_{i}^{\prime} b_{i}^{\prime \lambda},
$$

where $a_{i}^{\prime}=a_{i} \ln \left(b_{i} / b_{1}\right)$ and $b_{i}^{\prime}=b_{i} / b_{1}$. Assume that this expression is identically zero; then $\sum_{i=1}^{n} a_{i} b_{i}^{\lambda}=0$ must be a constant, and the claim follows. Assume that the derivative does not vanish for all $\lambda$. Now by the induction hypothesis, the derivative has at most $n-2$ zeros. But $y_{1}, \ldots, y_{k}$ are solutions to the above equation with multiplicities $t_{1}-1, \ldots, t_{k}-1$; also, by Rolle's theorem, the derivative has at least one root in each interval $\left(y_{i} ; y_{i+1}\right)$, and thus we obtain $k-1+\sum_{i=1}^{k} t_{i}-1$ solutions (counted with their multiplicities), which is greater than $n-2$; a contradiction. It follows that $\sum_{i=1}^{k} t_{i} \leq n-1$.

We continue with another problem, which is a part of Theorem 1.2.

Theorem 2.2. Let $P_{1}, \ldots, P_{n}$ be the vertices of a regular polygon, given a circle $\Gamma$, concentric to the circle circumscribed around $P_{1} P_{2} \ldots P_{n}$; then $\sum_{i=1}^{n} P P_{i}^{2 k}$ is independent of the position of $P \in \Gamma$ for $k \in\{1, \ldots, n-1\}$.

Proof. We use complex numbers. We may assume that the circumscribed circle around $P_{1} \ldots P_{n}$ is the unit circle and that the radius of $\Gamma$ is $R$. Let us assign to the vertices of the $n$-gon the complex numbers $\xi, \xi^{2}, \ldots, \xi^{n}$, where $\xi$ is a primitive 
$n$-th root of unity. We wish to prove that $\sum_{i=1}^{n}\left|x-\xi^{i}\right|^{2 k}=$ const for all $x$ with a fixed norm $R$ and all $k \in\{1, \ldots, n-1\}$. We have

$$
\sum_{i=1}^{n}\left|x-\xi^{i}\right|^{2 k}=\sum_{i=1}^{n}\left(x-\xi^{i}\right)^{k}\left(\overline{x-\xi^{i}}\right)^{k}=\sum_{i=1}^{n}\left(x-\xi^{i}\right)^{k}\left(\frac{R}{x}-\frac{1}{\xi^{i}}\right)^{k} .
$$

After multiplying, out we obtain

$$
\left(x-\xi^{i}\right)^{k}\left(\frac{R}{x}-\frac{1}{\xi^{i}}\right)^{k}=\sum_{j=-k}^{k} c_{j} \xi^{-i j} x^{j}=P_{i}(x)
$$

for all $x$ with $|x|=R$. We now have

$$
\sum_{i=1}^{n}\left|x-\xi^{i}\right|^{2 k}=\sum_{i=1}^{n} P_{i}(x)=\sum_{j=-k}^{k} \sum_{i=1}^{n} c_{j} \xi^{-i j} x^{j},
$$

but $\sum_{i=1}^{n} \xi^{-i j}=0$ for all $j$ except $j=m n$, where $m$ is an integer, so

$$
\sum_{i=1}^{n}\left|x-\xi^{i}\right|^{2 k}=n c_{0}
$$

Remark 2.3. One can prove that this is a characteristic property of the regular $n$-gon. Given $n$ different points in the plane $A_{1}, \ldots, A_{n}$ and a circle $\Gamma$, such that $\sum_{i=1}^{n} P A_{i}^{2 k}$ is independent of the position of $P$ on $\Gamma$ for every $k \in\{1, \ldots, n-1\}$, then these points are the vertices of a regular $n$-gon. It is conjectured that this remains true if the condition holds only for $k=2 n-2$, and this has been verified for $n=3$ and $n=4$, but the authors have no proof for higher values of $n$.

Proof of Theorem 1.2. We may assume $X \in \widehat{B_{1} M}$, where $M$ is the midpoint of $\operatorname{arc} \widehat{B_{1} B_{2}}$, because of symmetry.

After we position ourselves in a Cartesian coordinate system without loss of generality, we can assume that $P_{1}$ has coordinates $(1,0)$. Thus the coordinates of $P_{i}$ are $(\cos ((i-1) 2 \pi / n), \sin ((i-1) 2 \pi / n))$, and $X$ has coordinates $(a \cos x, a \sin x)$, where $x \in[0 ; 2 \pi / n]$.

We can now write the sum

$$
\begin{aligned}
\sum_{i=1}^{n}\left|P_{i} X\right|^{\lambda} & =\sum_{i=1}^{n}\left(\left(a \cos x-\cos \frac{(i-1) 2 \pi}{n}\right)^{2}+\left(a \sin x-\sin \frac{(i-1) 2 \pi}{n}\right)^{2}\right)^{\lambda / 2} \\
& =F(x, \lambda) .
\end{aligned}
$$

We differentiate this with respect to $x$ to obtain

$$
\frac{\partial F(x, \lambda)}{\partial x}=\sum_{i=1}^{n} \lambda\left|P_{i} X\right|^{\lambda-1} \frac{d\left|P_{i} X\right|}{d x} .
$$


The partial derivative exists for $x \in(0 ; 2 \pi / n)$. Now fix $x$ and consider this as a function of $\lambda$. As we are interested only in the sign of the derivative, we can consider only

$$
\sum_{i=1}^{n}\left|P_{i} X\right|^{\lambda-1} \frac{d\left|P_{i} X\right|}{d x}
$$

for $\lambda \neq 0$.

As proved earlier, $F(x, \lambda)$ is constant for $\lambda=2,4, \ldots, 2 n-2$, so $\partial F(x, \lambda) / \partial x$ vanishes for these values of $\lambda$. But from Lemma 2.1, this expression is either identically zero or has at most $n-1$ solutions for $\lambda$, counted with their multiplicities.

We prove that this expression as a function of $\lambda$ is not identically zero for fixed $x \in(0 ; 2 \pi / n)$. For the sake of contradiction, assume otherwise. Let $x \in(0 ; 2 \pi / n)$. It is easy to see that for the point $X$ corresponding to this $x$, the distances $\left|P_{i} X\right|$ are all different. Now take $i$ such that $\left|P_{i} X\right|$ is maximized subject to the condition that $d\left|P_{i} X\right| / d x$ is nonzero. If $\left|P_{i} X\right|>1$, then

$$
\lim _{\lambda \rightarrow \infty}\left|\frac{\partial F(x, \lambda)}{\partial x}\right|=\infty \text {. }
$$

On the other hand, if $\left|P_{i} X\right| \leq 1$, choose $i$ instead so that $\left|P_{i} X\right|$ is minimized subject to the condition that $d\left|P_{i} X\right| / d x$ is nonzero. In this case $\left|P_{i} X\right|<1$; otherwise there must be two distances $P_{i} X$ that are equal (or else $n-1$ among the $d\left|P_{i} X\right| / d x$ vanish, which is not possible). Indeed, as $X$ describes the arcs $\widehat{P_{i}^{\prime} P_{i}^{\prime \prime}}$ and $\widehat{P_{i}^{\prime \prime} P_{i}^{\prime}}$, where $P_{i}^{\prime}, P_{i}^{\prime \prime}$ are the two intersections of $\Gamma$ with the line through $O$ and $P_{i}$, the distance $P_{i} X$ increases along one arc and decreases along the other. It is obvious that $P_{i}^{\prime}$ and $P_{i}^{\prime \prime}$ either coincide with some of $B_{i}$ or are midpoints of some arc between consecutive vertices of $B_{1} \ldots B_{n}$.

Again considering $\lim _{\lambda \rightarrow-\infty}|\partial F(x, \lambda) / \partial x|=\infty$, we obtain the desired result.

As mentioned, for a fixed $x, \partial F(x, \lambda) / \partial x$ vanishes for every $\lambda=0,2,4, \ldots$, $2 n-2$, and it follows that these are all the solutions for $\lambda$ and that each of them - except possibly $\lambda=0$ - must have multiplicity one. Also the derivative changes sign at $\lambda=0$. For a fixed $x$, therefore, $\partial F(x, \lambda) / \partial x$ changes sign at $\lambda=0,2,4, \ldots, 2 n-2$. Now assume that for some $\lambda_{0} \neq 0,2,4, \ldots, 2 n-2$, there exist $y$ and $z$ in the interval $(0 ; 2 \pi / n)$ such that

$$
\frac{\partial F\left(y, \lambda_{0}\right)}{\partial x} \frac{\partial F\left(z, \lambda_{0}\right)}{\partial x}<0 ;
$$

then since $\partial F\left(x, \lambda_{0}\right) / \partial x$ is a continuous function of $x$, there is $t \in(0 ; 2 \pi / n)$ such that $\partial F\left(t, \lambda_{0}\right) / \partial x=0$, and it follows that $\partial F(t, \lambda) / \partial x=0$ for all $\lambda$, which is a contradiction. Hence the derivative

$$
\frac{\partial F(x, \lambda)}{\partial x}
$$


does not change when $x \in(0 ; 2 \pi / n)$ for fixed $\lambda$; also, for every fixed $x \in(0,2 \pi / n)$ it changes sign at $\lambda=0,2, \ldots, 2 n-2$. Thus, since $F(x, \lambda)$ is a continuous function of $x$, we have shown that the minimum and maximum of that function for $x \in$ $[0 ; 2 \pi / n]$ are obtained when $x=0$, or $x=2 \pi / n$.

Now consider

$$
\lim _{\lambda \rightarrow \infty} \frac{F(0, \lambda)}{F(2 \pi / n, \lambda)}
$$

Assume that $n$ is even; then $\left|B_{1} P_{n / 2+1}\right|>\left|M P_{i}\right|$ for every $i$, and the limit is $\infty$.

Assume that $n$ is odd; then $\left|M P_{\lceil n / 2\rceil}\right|>\mid B_{1} P_{i}$ for every $i$, and the above limit becomes 0 . This proves part (3) of Theorem 1.2. Now, taking into account parity and the above observations for the intervals in which $\partial F(x, \lambda) / \partial x$ changes sign, the conclusion of the theorem easily follows.

Remark 2.4. When $\Gamma$ is the circumcircle of the regular polygon, Theorem 1.2(1) is easily proved by the observation that each of the functions $M P_{i}^{\lambda}+M P_{n+1-i}^{\lambda}$ is concave.

\section{The case for three base points (proof of Theorem 1.1)}

Proof of the case $\lambda<\mathbf{0}$. We now consider the case $\lambda<0$.

Let $\angle C=\max \{\angle A, \angle B, \angle C\}$, and let $M$ be the midpoint of the smaller arc $\widehat{A B}$. We prove that $f(M, \lambda) \leq 2+2^{\lambda}$. We consider two cases:

Case 1: $\angle C \geq \pi / 2$. Then the maximum of the function $M A^{\lambda}+M B^{\lambda}+M C^{\lambda}$ when $C$ travels along the smaller arc $\widehat{A B}$ is achieved when $C \equiv A$ or when $C \equiv B$, since $M D>M A=M B$ for any point $D$ on the smaller $\operatorname{arc} \widehat{A B}$. Now we have $f(M, \lambda)=3 M A^{\lambda} \leq 3 \sqrt{2}^{\lambda}<2^{\lambda}+2$.

Case 2: $\angle C=x<\pi / 2$. Then $\angle C \in[\pi / 3 ; \pi / 2)$. Now let $C^{\prime}$ and $C^{\prime \prime}$ be the points for which $\angle A B C^{\prime}$ and $\angle B A C^{\prime \prime}$ equal $x$. It is easy to see that $C$ belongs to the smaller arc $\widehat{C^{\prime} C^{\prime \prime}}$, since $\angle C$ is the largest angle of the triangle. Also, the maximum of $f(M, \lambda)$ when $C$ belongs to the arc $\widehat{C^{\prime} C^{\prime \prime}}$ is obtained exactly when $C \equiv C^{\prime}$ or $C \equiv C^{\prime \prime}$, since $M C^{\prime \prime}=M C^{\prime} \leq M C$ for every $C$ on $\widehat{C^{\prime} C^{\prime \prime}}$. Without loss of generality we can assume that $C \equiv C^{\prime}$. Then we can write

$$
f(M, \lambda)=2\left(2 \sin \frac{1}{2} x\right)^{\lambda}+\left(2 \sin \frac{3}{2} x\right)^{\lambda}=2^{\lambda}\left(2 \sin ^{\lambda} \frac{1}{2} x+\sin ^{\lambda} \frac{3}{2} x\right)=F(x, \lambda) .
$$

We differentiate with respect to $x$ to get

$$
\frac{\partial F(x, \lambda)}{\partial x}=\lambda 2^{\lambda}\left(\sin ^{\lambda-1} \frac{1}{2} x \cos \frac{1}{2} x+\frac{3}{2} \sin ^{\lambda-1} \frac{3}{2} x \cos \frac{3}{2} x\right) .
$$

Clearly, both $\sin ^{\lambda-1} \frac{1}{2} x \cos \frac{1}{2} x$ and $\frac{3}{2} \sin ^{\lambda-1} \frac{3}{2} x \cos \frac{3}{2} x$ are decreasing functions in the interval $[\pi / 3 ; \pi / 2)$, since $\lambda<0$. 
Then $\partial F(x, \lambda) / \partial x$ is an increasing function of $x$ in this interval since $\lambda<0$, and hence $F(x, \lambda)$ is a convex function of $x \in[\pi / 3 ; \pi / 2)$ if $\lambda<0$.

From here it follows that

$$
\begin{aligned}
\sup _{x \in[\pi / 3 ; \pi / 2)} F(x, \lambda) & =\max \left\{F(\pi / 3, \lambda), \lim _{x \rightarrow \pi / 2} F(x, \lambda)\right\} \\
& =\max \left\{2+2^{\lambda}, 3 \sqrt{2}^{\lambda}\right\},
\end{aligned}
$$

since $F(x, \lambda)$ is continuous in the interval $[\pi / 3 ; \pi / 2]$. Then $\max \left\{2+2^{\lambda}, 3 \sqrt{2}^{\lambda}\right\}=$ $2+2^{\lambda}$, and this bound is achievable only for $A B C$ an equilateral triangle; for all other configurations of the points $A B C$, the function $M A^{\lambda}+M B^{\lambda}+M C^{\lambda}$ is less than $2+2^{\lambda}$ for the specified point $M$.

Now using Theorem 1.2, we get that the minimum of $M A^{\lambda}+M B^{\lambda}+M C^{\lambda}$ is obtained when $M$ bisects the arc between consecutive vertices of the triangle, and in this case we have $M A^{\lambda}+M B^{\lambda}+M C^{\lambda}=2+2^{\lambda}$. This concludes the proof.

Remark 3.1. This case can also be proved using the main approach of [Ambrus and Ball 2011], which is based on Lemma 1 of that reference, namely, the fact that the local minima on each of the arcs between consecutive base points must be equal for all $\lambda<0$. In the case of only three points, one can obtain that the equilateral triangle is indeed the extremal case. Assume otherwise. Clearly it is not possible for all of the local minima to be equal when two of the points are closer than $\sqrt{2}$. Assume now that $C$ is not the midpoint of the arc $\widehat{A B}$. Consider the function

$$
f_{1}=|M A|^{\lambda}+|M B|^{\lambda}+\left|M C_{1}\right|^{\lambda},
$$

where $C_{1}$ is the midpoint of $\widehat{A B}$. We may assume that $C$ belongs to the shorter arc $C_{1} B$. By symmetry, the local minima of $f_{1}$ are equal on the short arcs $\widehat{A C}_{1}$ and $\widehat{B C}_{1}$. Now we have $\angle C_{1} O C \leq \pi / 4$. Thus $f>f_{1}$ on $\widehat{A C}_{2}$ and $f<f_{1}$ on $\widehat{B C}_{2}$, where $C_{2}$ is the midpoint of the arc $\widehat{C C}_{1}$. From here we obtain that the local minima of $f$ cannot be equal on the shorter arcs $\widehat{A C}$ and $\widehat{B C} .{ }^{1}$

Proof of the case $\lambda>2$. We first prove that for every three points $A, B$ and $C$ on the unit circle there exists a point $M$ also on the unit circle, such that $f(M, \lambda) \geq$ $\max \left\{2+2^{\lambda}, 2(\sqrt{3})^{\lambda}\right\}$.

Let $A B=\min \{A B, B C, C A\}$; now let the bisector of $A B$ intersect the larger $\operatorname{arc} A B$ at $M^{\prime}$. Then $\angle B A M^{\prime}=\angle A B M^{\prime}=x$ and $\pi / 3 \leq x<\pi / 2$. Now by the sine rule, $B M^{\prime}=A M^{\prime}=2 \sin x \geq \sqrt{3}$, and we have $f\left(M^{\prime}, \lambda\right) \geq 2(\sqrt{3})^{\lambda}$ with equality only if $x=\pi / 3$, or, equivalently, if $A B C$ is an equilateral triangle.

It remains to prove that for every triangle there is a point $M^{\prime}$ such that $f\left(M^{\prime}, \lambda\right) \geq$ $2+2^{\lambda}$. We consider two cases - when $A B C$ is obtuse-angled and when it is acuteangled.

${ }^{1}$ The authors thank the referee for suggesting this approach. 
Case 1: Let $\angle C=\max \{\angle A, \angle B, \angle C\} \geq \pi / 2$, and let $O$ be the center of $\Gamma$. Let $M^{\prime}=C O \cap \Gamma$. We have $C M^{\prime}=2$ and $f\left(M^{\prime}, \lambda\right)=2^{\lambda}+B M^{\prime \lambda}+A M^{\prime \lambda}$. Now $\angle B A M^{\prime}, \angle A B M^{\prime} \leq \pi / 2$ and $\angle B A M^{\prime}+\angle A B M^{\prime} \geq \pi / 2$.

We have $\pi / 4 \leq \max \left\{\angle B A M^{\prime}, \angle A B M^{\prime}\right\} \leq \pi / 2$, and so

$$
B M^{\prime \lambda}+A M^{\prime \lambda}>(2.1 / \sqrt{2})^{\lambda} \geq 2
$$

as $\lambda \geq 2$, so $f\left(M^{\prime}, \lambda\right)>2+2^{\lambda}$. And this bound cannot be achieved for an obtuseangled triangle.

Case 2: Let $c=\angle C=\max \{\angle A, \angle B, \angle C\}<\pi / 2$. Now $M^{\prime}=C O \cap \Gamma$. We have $C M^{\prime}=2$ and $f\left(M^{\prime}, \lambda\right)=2^{\lambda}+B M^{\prime \lambda}+A M^{\prime \lambda}$. We prove that $B M^{\prime \lambda}+A M^{\prime \lambda} \geq 2$. Let $\angle A C M^{\prime}=x$. By the sine rule, $B M^{\prime \lambda}+A M^{\prime \lambda}=(2 \sin x)^{\lambda}+(2 \sin c-x)^{\lambda}=$ $f_{1}(x, \lambda)$. We prove that $\sin ^{\lambda} x+\sin ^{\lambda}(c-x)>2 \sin ^{\lambda}(c / 2)$. We have

$$
\begin{aligned}
\frac{\partial f_{1}(x, \lambda)}{\partial x} & =\lambda 2^{\lambda}\left(\sin ^{\lambda-1} x \cos x-\sin ^{\lambda-1}(c-x) \cos (c-x)\right) \\
& =\lambda 2^{\lambda-1}\left(\sin ^{\lambda-2} x \sin 2 x-\sin ^{\lambda-2}(c-x) \sin (2 c-2 x)\right) .
\end{aligned}
$$

It is now easy to see that for $x \in\left[0 ; c / 2\right.$ ), we have $\sin ^{\lambda-2} x<\sin ^{\lambda-2}(c-x$ ) (for $\lambda \geq 2)$ and $\sin 2 x<\sin (2 c-2 x)$, and so

$$
\frac{\partial f_{1}(x, \lambda)}{\partial x}<0
$$

With similar arguments it follows that $\frac{\partial f_{1}(x, \lambda)}{\partial x}=0$ for $x=\frac{c}{2}$, and $\frac{\partial f_{1}(x, \lambda)}{\partial x}>0$ for $x \in(c / 2 ; c]$.

Then $\min _{x \in[0 ; c]}\left(f_{1}(x, \lambda)+f_{1}(c-x, \lambda)\right)=2 f_{1}(c / 2, \lambda) \geq 2 f_{1}(\pi / 6, \lambda)=2$. Equality holds if and only if $A B C$ is equilateral.

We have $2+2^{\lambda}=2 \sqrt{3}^{\lambda}$ for $\lambda \in\{2,4\} ; 2+2^{\lambda}<2 \sqrt{3}^{\lambda}$ for $\lambda \in(2 ; 4)$; and $2+2^{\lambda}>2 \sqrt{3}^{\lambda}$ for $\lambda>4$. We prove that for $A B C$ an equilateral triangle, those bounds are sharp.

Again using Theorem 1.2, we get:

(1) When $\lambda \in[2 ; 4]$, the maximum of $M A^{\lambda}+M B^{\lambda}+M C^{\lambda}$ is achieved when $M$ coincides with one of $A, B, C$ and is equal to $2 \sqrt{3}^{\lambda}$.

(2) When $\lambda>4$, the maximum of $M A^{\lambda}+M B^{\lambda}+M C^{\lambda}$ is achieved when $M$ bisects the arc between consecutive vertices of the triangle $A B C$ and is equal to $2+2^{\lambda}$.

These bounds are sharp.

We also have the minimum of $f(M, \lambda)$ when $A B C$ is an equilateral triangle, that is, when $\lambda \in[2,4]$, we have $\min f(M, \lambda)=2+2^{\lambda}$; and $\min f(M, \lambda)=2 \sqrt{3}^{\lambda}$ when $\lambda>4$. This concludes the proof. 
Proof of the case $\lambda \in[0 ; 2]$. This case is proved in [Stolarsky 1975], but we give a new, independent proof.

We now prove that for every three points $A, B, C$ on the unit circle and a real number $\lambda \in(0 ; 2)$, there exists a point $M$ also on the unit circle, such that $f(M, \lambda)=M A^{\lambda}+M B^{\lambda}+M C^{\lambda} \geq 2+2^{\lambda}$; and this bound is sharp. It is only achievable when $A, B$ and $C$ are the vertices of a equilateral triangle.

Again let $\angle C=\max \{\angle A, \angle B, \angle C\}=x$. As before, it is easy to see that when $\angle C \geq \pi / 2$ (with $M$ chosen as the midpoint of the arc $\widehat{A B}$ ), we have

$$
f(M, \lambda) \geq 3 \sqrt{2}^{\lambda} \geq 2+2^{\lambda}
$$

for $\lambda \in[0 ; 2]$. When $\lambda \in\{0,2\}$, we have that $M A^{\lambda}+M B^{\lambda}+M C^{\lambda}$ is constant. We can assume that the triangle $A B C$ is acute-angled. Then $\angle C \in[\pi / 3 ; \pi / 2]$, and again let $M$ be the midpoint of the arc $\widehat{A B}$. Now let $C^{\prime}$ and $C^{\prime \prime}$ be the points for which $\angle A B C^{\prime}$ and $\angle B A C^{\prime \prime}$ respectively equal $x$. It is easy to see that $C$ belongs to the smaller arc $\widehat{C^{\prime} C^{\prime \prime}}$, since $C$ is the largest angle of the triangle. It is also easy to see that the minimum of $f(M, \lambda)$ when $C$ belongs to the arc $\widehat{C^{\prime} C^{\prime \prime}}$ is obtained exactly when $C \equiv C^{\prime}$ or $C \equiv C^{\prime \prime}$, since $M C^{\prime \prime}=M C^{\prime} \leq M C$ for every $C$ on $\widehat{C^{\prime} C^{\prime \prime}}$.

Let $\angle A=x$. Using the sine rule, we now get

$$
f(M, \lambda)=M A^{\lambda}+M B^{\lambda}+M C^{\lambda}=2\left(2 \sin \frac{1}{2} x\right)^{\lambda}+\left(\sin \frac{3}{2} x\right)^{\lambda}=F(x, \lambda) .
$$

We prove that if $x \in[\pi / 3 ; \pi / 2]$, then $F(x, \lambda) \geq 2+2^{\lambda}$.

We consider two cases:

Case 1: $\lambda \in(0 ; 1)$. After differentiating with respect to $x$, we get

$$
\frac{\partial F(x, \lambda)}{\partial x}=\lambda 2^{\lambda}\left(\sin ^{\lambda-1} \frac{1}{2} x \cos \frac{1}{2} x+\frac{3}{2} \sin ^{\lambda-1} \frac{3}{2} x \cos \frac{3}{2} x\right) .
$$

It is now easy to see that both $\sin ^{\lambda-1} \frac{1}{2} x \cos \frac{1}{2} x$ and $\sin ^{\lambda-1} \frac{3}{2} x \cos \frac{3}{2} x$ are decreasing functions as $\lambda-1<0$. Then $F(x, \lambda)$ is a concave function of $x$ when $\lambda \in(0 ; 1)$. It follows that

$$
\begin{aligned}
\min _{x \in[\pi / 3 ; \pi / 2)} F(x, \lambda) & =\min \left\{F(\pi / 3, \lambda), \lim _{x \rightarrow \pi / 2} F(x, \lambda)\right\} \\
& =F(\pi / 3 ; \lambda)=2+2^{\lambda},
\end{aligned}
$$

and for every $x \neq \pi / 3$ we have $F(x, \lambda)>2+2^{\lambda}$. We shall later prove that when $A, B, C$ are the vertices of an equilateral triangle this bound is sharp.

$\underline{\text { Case 2: }} \lambda \in[1 ; 2)$. Let $C O \cap \Gamma=M$. We shall prove that for the point $M$ we have $A M^{\lambda}+B M^{\lambda}+C M^{\lambda} \geq 2+2^{\lambda}$. We have $C M=2^{\lambda}$. We only need to prove that $B M+C M \geq 2$, since

$$
B M^{\lambda}+A M^{\lambda} \geq 2\left(\frac{A M+B M}{2}\right)^{\lambda} .
$$


Lemma 3.2. Let $A, B$ and $C$ be points on the unit circle $\Gamma$ with center $O$. Assume $\angle C=\max \{\angle A, \angle B, \angle C\}$ and $M=C O \cap \Gamma$ then $M A+M B \geq 2$.

Proof. We have

$$
M A+M B=2(\sin x+\sin (c-x))=: f(x),
$$

where $x=\angle M A C$ and $c=\pi-\angle A C B<\pi / 2$. Then $f^{\prime}(x)=2(\cos x-\cos (c-x))$; thus $f^{\prime}(x)>0$ for $x \in[0 ; c / 2)$, while $f^{\prime}(x)=0$ for $x=c / 2$ and $f^{\prime}(x)<0$ for $x \in(c / 2 ; c]$.

Now let $C^{\prime}$ and $C^{\prime \prime}$ be the points for which $\angle A B C^{\prime}$ and $\angle B A C^{\prime \prime}$ respectively equal $x$. It is easy to see that $C$ belongs to the smaller arc $\widehat{C^{\prime} C^{\prime \prime}}$ as $C$ is the largest angle of the triangle. The minimum min $M A^{\lambda}+M B^{\lambda}$ is obtained when $M O \cap \Gamma=C^{\prime}$ or $M O \cap \Gamma=C^{\prime \prime}$ as $f(x)$ is concave. Now $\angle A B C=\angle B C A=\gamma$. Then $M A+M B=2(\sin (\pi / 2-\gamma)+\sin (2 \gamma-\pi / 2))=\cos \gamma-\cos 2 \gamma=f_{1}(\gamma)$. Differentiating $f_{1}(\gamma)$ we get $f_{1}^{\prime}(\gamma)=2 \sin 2 \gamma-\sin \gamma$ which is a decreasing function of $\gamma \in[\pi / 3 ; \pi / 2)$. This gives us that $f_{1}(\gamma)$ is a concave function when $\gamma \in$ $[\pi / 3 ; \pi / 2)$, and it follows that

$$
\min f_{1}(\gamma)=\min _{\gamma \in[\pi / 3 ; \pi / 2)}\left\{f_{1}(\pi / 3), \lim _{x \rightarrow \pi / 2} f_{1}(x)\right\}=2 .
$$

Now

$$
B M^{\lambda}+A M^{\lambda} \geq 2\left(\frac{A M+B M}{2}\right)^{\lambda} \geq 2,
$$

with equality only when $A M=B M=1$ which is possible only when $A, B$ and $C$ are the vertices of an equilateral triangle.

In such a way we obtain that when $\lambda \in(0 ; 2)$ there exists a point $M$ on the unit circle, such that $M A^{\lambda}+M B^{\lambda}+M C^{\lambda} \geq 2+2^{\lambda}$ and this bound is achievable only if $A, B$ and $C$ are the vertices of an equilateral triangle.

Now using again the result of Theorem 1.2 one easily obtains that the maximum of $M A^{\lambda}+M B^{\lambda}+M C^{\lambda}$ is obtained when $M$ is the midpoint of one of the arcs between consecutive vertices and it indeed equals $2+2^{\lambda}$.

\section{Acknowledgement}

The authors would like to thank the referee for his comments and remarks, which helped improve the clarity of the paper.

\section{References}

[Ambrus and Ball 2011] G. Ambrus and K. Ball, "Chebyshev constants for the unit circle", preprint, 2011. arXiv $1006.5153 \mathrm{v} 2$

[Mushkarov 1982] O. Mushkarov, “Тригонометрични полиноми и правилни многоъгълници”, Matematika (Sofia) 8 (1982), 11-16. Available online as a supplement to this paper. An 
English summary by J. Tabov can be found in "Sums of powers of distances in regular polygons and polyhedra", James Cook Math. Notes 5:50 (1989), page 5209, available at www.maths.ed.ac.uk/ cook/iss_no50.PDF.

[Stolarsky 1975] K. B. Stolarsky, "The sum of the distances to certain pointsets on the unit circle", Pacific J. Math. 59:1 (1975), 241-251. MR 52 \#9084 Zbl 0313.52006

Received December 27, 2010. Revised July 16, 2011.

NiKOLAI NiKOLOV

INSTITUTE OF MATHEMATICS AND INFORMATICS

BULGARIAN ACADEMY OF SCIENCES

ACAD. G. BONCHEV 8, 1113 SOFIA

BULGARIA

nik@math.bas.bg

RAFAEL RAFAILOV

SOFIA High SCHOOL OF Mathematics

ISKAR 61, 1000 SOFIA

BULGARIA

rafael.rafailov@yahoo.com 


\title{
PACIFIC JOURNAL OF MATHEMATICS
}

\author{
http://www.pjmath.org \\ Founded in 1951 by
}

E. F. Beckenbach (1906-1982) and F. Wolf (1904-1989)

\section{EDITORS}

V. S. Varadarajan (Managing Editor)

Department of Mathematics

University of California

Los Angeles, CA 90095-1555

pacific@math.ucla.edu

Vyjayanthi Chari

Department of Mathematics

University of California

Riverside, CA 92521-0135

chari@math.ucr.edu

\section{Robert Finn}

Department of Mathematics Stanford University

Stanford, CA 94305-2125

finn@math.stanford.edu

Kefeng Liu

Department of Mathematics

University of California

Los Angeles, CA 90095-1555

liu@math.ucla.edu
Darren Long

Department of Mathematics

University of California

Santa Barbara, CA 93106-3080

long@math.ucsb.edu

Jiang-Hua Lu

Department of Mathematics

The University of Hong Kong

Pokfulam Rd., Hong Kong jhlu@maths.hku.hk

Alexander Merkurjev

Department of Mathematics University of California

Los Angeles, CA 90095-1555 merkurev@math.ucla.edu
Sorin Popa

Department of Mathematics

University of California

Los Angeles, CA 90095-1555

popa@math.ucla.edu

Jie Qing

Department of Mathematics

University of California

Santa Cruz, CA 95064

qing@ cats.ucsc.edu

Jonathan Rogawski

Department of Mathematics

University of California

Los Angeles, CA 90095-1555

jonr@math.ucla.edu

\section{PRODUCTION}

pacific@math.berkeley.edu

Silvio Levy, Scientific Editor Matthew Cargo, Senior Production Editor

ACADEMIA SINICA, TAIPEI

CALIFORNIA INST. OF TECHNOLOGY

INST. DE MATEMÁTICA PURA E APLICADA

KEIO UNIVERSITY

MATH. SCIENCES RESEARCH INSTITUTE

NEW MEXICO STATE UNIV.

OREGON STATE UNIV.

\section{SUPPORTING INSTITUTIONS}

STANFORD UNIVERSITY
UNIV. OF BRITISH COLUMBIA
UNIV. OF CALIFORNIA, BERKELEY
UNIV. OF CALIFORNIA, DAVIS
UNIV. OF CALIFORNIA, LOS ANGELES
UNIV. OF CALIFORNIA, RIVERSIDE
UNIV. OF CALIFORNIA, SAN DIEGO
UNIV. OF CALIF., SANTA BARBARA

UNIV. OF CALIF., SANTA CRUZ

UNIV. OF MONTANA

UNIV. OF OREGON

UNIV. OF SOUTHERN CALIFORNIA

UNIV. OF UTAH

UNIV. OF WASHINGTON

WASHINGTON STATE UNIVERSITY

These supporting institutions contribute to the cost of publication of this Journal, but they are not owners or publishers and have no responsibility for its contents or policies.

See inside back cover or www.pjmath.org for submission instructions.

The subscription price for 2011 is US \$420/year for the electronic version, and \$485/year for print and electronic.

Subscriptions, requests for back issues from the last three years and changes of subscribers address should be sent to Pacific Journal of Mathematics, P.O. Box 4163, Berkeley, CA 94704-0163, U.S.A. Prior back issues are obtainable from Periodicals Service Company, 11 Main Street, Germantown, NY 12526-5635. The Pacific Journal of Mathematics is indexed by Mathematical Reviews, Zentralblatt MATH, PASCAL CNRS Index, Referativnyi Zhurnal, Current Mathematical Publications and the Science Citation Index.

The Pacific Journal of Mathematics (ISSN 0030-8730) at the University of California, c/o Department of Mathematics, 969 Evans Hall, Berkeley, CA 94720-3840, is published monthly except July and August. Periodical rate postage paid at Berkeley, CA 94704, and additional mailing offices. POSTMASTER: send address changes to Pacific Journal of Mathematics, P.O. Box 4163, Berkeley, CA 94704-0163.

PJM peer review and production are managed by EditFLOW ${ }^{\mathrm{TM}}$ from Mathematical Sciences Publishers.

PUBLISHED BY PACIFIC JOURNAL OF MATHEMATICS

at the University of California, Berkeley 94720-3840

A NON-PROFIT CORPORATION

Typeset in IATEX

Copyright $(2011$ by Pacific Journal of Mathematics 


\title{
PACIFIC JOURNAL OF MATHEMATICS
}

\author{
Volume $253 \quad$ No. $1 \quad$ September 2011
}

Singularities of the projective dual variety

ROLAND ABUAF

Eigenvalue estimates for hypersurfaces in $\mathbb{H}^{m} \times \mathbb{R}$ and applications

Pierre BÉRARD, Philippe CASTILlon and Marcos CaVAlCante

Conformal Invariants associated to a measure: Conformally covariant operators

Sun-Yung A. Chang, Matthew J. Gursky and Paul Yang

Compact symmetric spaces, triangular factorization, and Cayley coordinates

DEREK HABERMAS

Automorphisms of the three-torus preserving a genus-three Heegaard splitting

JESSE JOHNSON

The rationality problem for purely monomial group actions

HiDETAKA KiTAYAMA

On a Neumann problem with $p$-Laplacian and noncoercive resonant nonlinearity

Salvatore A. Marano and Nikolaos S. Papageorgiou

Minimal ramification in nilpotent extensions

NADYA MARKIN and STEPHEN V. ULLOM

Regularity of weakly harmonic maps from a Finsler surface into an $n$-sphere

XIAOHUAN MO and LIANG ZHAO

On the sum of powered distances to certain sets of points on the circle

NikOlai NikOlOV and RAFAEL RAFAilov

Formal geometric quantization II

PAUL-ÉMILE PARADAN

Embedded constant-curvature curves on convex surfaces

HAROLD ROSENBERG and MATTHIAS SCHNEIDER

A topological construction for all two-row Springer varieties 